\title{
Quantum Machine Learning for Data Classification
}

\section{Quantum machine-learning techniques speed up the task of classifying data delivered by a small network of quantum sensors.}

\author{
By Seth Lloyd
}

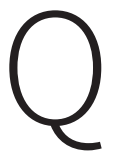
uantum technologies are growing at great pace, driven by the interplay between academia, government, startups, and large companies such as Google, IBM, Microsoft, and Amazon. Quantum communication systems, including quantum cryptographic networks, have already been deployed on large scales; quantum metrology schemes, such as atomic clocks, offer the state of the art for high-precision measurements; and quantum computers are entering an early industrial era. Yet the "quantum jungle" of available devices and protocols remains hard to navigate, and researchers still need to work on identifying the most promising paths to

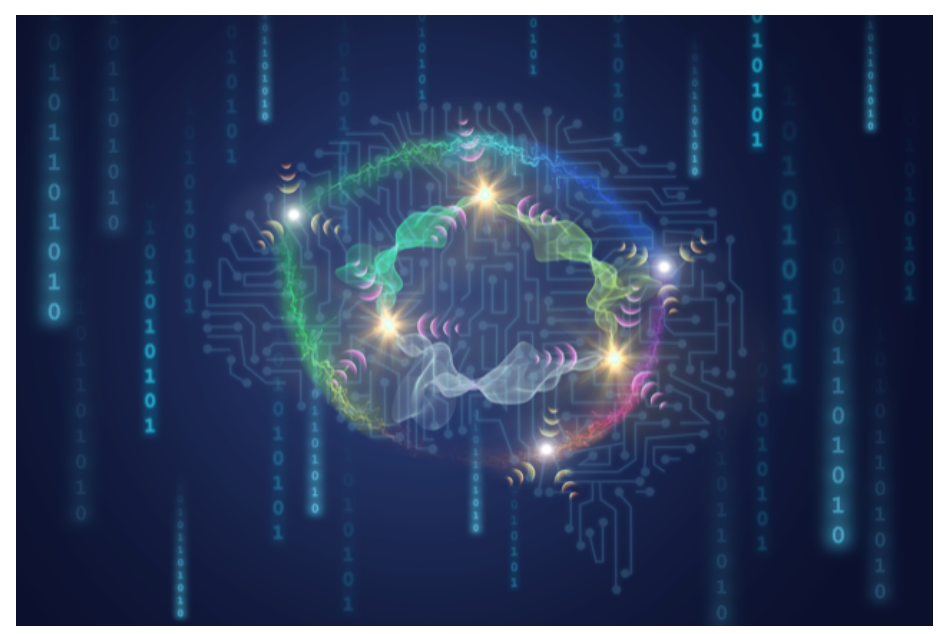

Figure 1: Artistic rendition of the quantum-enhanced classification of data from a network of entangled sensors. The work of Zhang, Zhuang, and co-workers suggests that this quantum machine-learning approach holds promise to benefit real-world data-classification applications.

Credit: Sondii Media quantum technologies that can be societally useful. Now, a collaboration between two teams at the University of Arizona-led by Zheshen Zhang and by Quntao Zhuang, respectively-shows that quantum entanglement can provide a quantifiable advantage to data classification, a task relevant for imaging and navigation [1]. Using a machine-learning algorithm, the team classifies data from a network of entangled sensors (Fig. 1). By comparing their scheme with one using classical data processing, they show that entanglement can boost both the accuracy and the speed of classification. The work paves the way for a broad range of quantum-enhanced classification methods that could be enabled by near-future quantum technologies.

There are good reasons to be optimistic about the future of quantum computers. But building a universal, fault-tolerant quantum computer-one that can correct errors deriving from imperfections in its operations or from environmental disturbances-is a daunting task. Fault-tolerant quantum computers that can be scaled up to solve meaningful problems are a decade away (plus or minus infinity, given the incertitude inherent in such predictions). In the meantime, however, the field of quantum simulation is forging ahead. Devices known as variational quantum eigensolvers are showing promise for solving hard problems in quantum chemistry [2,3] and for performing data-classification tasks [4]. The power of these quantum devices can be further boosted by incorporating techniques borrowed from the emerging field of machine learning. But can this potential be realized with the quantum hardware that will be available in the near term, that is, noisy, intermediate-scale quantum (NISQ) devices [5]? And can quantum machine-learning algorithms provide a true 
advantage over the powerful, classical machine-learning methods that are already available?

The work of Zhang, Zhuang, and co-workers explores a route for quantum-enhanced data processing that derives from the marriage of quantum machine learning with the most established quantum technologies: quantum sensing and metrology. A well-known example of such technologies is the quantum logic clock first developed by Nobel laureate David Wineland and colleagues [6]. Using methods of quantum computation, including the manipulation of entanglement, this type of clock achieves unprecedented precision in the measurement of time - it is, by many criteria, the most accurate measurement instrument ever constructed by humans. Related techniques have advanced measurements of other quantities (electric and magnetic fields, mass, acceleration, and more), approaching precision levels close to the ultimate limits set by the laws of quantum mechanics.

Combining techniques from the mature field of quantum metrology with techniques from quantum computing is thus especially promising. Recent theoretical and experimental work has shown the potential of using quantum machine-learning techniques to directly process "quantum data" acquired by quantum measurement and sensing devices, optimizing tasks such as measurement, discrimination, and data classification. Such direct processing has advantages compared with processing classical data using quantum techniques, which requires an interface called a quantum random access memory (qRAM), which loads classical data into a quantum computer [7]. This technology, however, is still in its infancy.

The work of Zhang's and Zhuang's teams focuses on a particularly intriguing case-a network of quantum sensors. The use of quantum information processing techniques to combine and analyze the quantum outputs of multiple sensors holds tremendous promise for realizing a quantum advantage. The potential gain stems from a fundamental feature of quantum metrology: The advantage provided by the coherent processing of sensor data scales as the square root of the dimension of the so-called Hilbert space that represents the quantum states sensed by the network. Since the dimension of that Hilbert space scales exponentially with the number of analyzed states, the quantum advantage for a quantum sensor network scales exponentially with the number of sensors.
The teams perform the first experimental study that applies quantum machine learning to quantum sensor networks, providing a compelling indication of a quantum advantage. The authors construct a sensor network that generates entangled states and encodes such states in radio-frequency signals. They then design a quantum protocol to classify these signals based on certain features of their amplitude-phase relationship. Such a protocol, dubbed supervised learning, assisted by an entangled quantum network uses techniques of variational optimization (analogous to the training of a deep neural network) to identify an optimal quantum measurement for analyzing the states and classifying the quantum data.

The authors investigate a framework that may be generalized to data-classification problems in many other physical domains. They analyze an experimental configuration in which a classical signal generates a quantum state in the quantum sensors. They then compare the optimal signal-discriminating measurements for two cases. In the first, the states generated by the quantum sensors are measured individually, and the results of the measurements are processed classically. In the second, the states generated by the sensors are processed by a quantum network that performs entangling operations between the states generated by different sensors. In both cases, the measurements are optimized by variational techniques to minimize the classification error using the established support vector machines (SVM) method. SVM classifies data on the quantum states by finding optimal "hyperplanes" that separate the states in a vector space. The researchers find that the quantum SVM provides a significant reduction in the error rate of classification compared with the error rate achieved with a classical SVM.

This first experimental demonstration is performed with just three quantum sensors-a tiny "quantum step" for the tiny "quantum feet" offered by current technologies. But the method can readily be extended to higher numbers of sensors and, given the exponential scaling, holds the promise of dramatically enhancing the power of large sensor networks. Importantly, such enhancement can be obtained with quantum information processors containing hundreds or thousands of quantum logic gates-those that might be available in the near term.

Zhang, Zhuang, and co-workers chose to explore the quantum 
jungle by following a path that runs at the border between quantum sensing and quantum machine learning. The beautiful "quantum fruits" they discovered along the way show that this path deserves further exploration.

Seth Lloyd: Massachusetts Institute of Technology, Cambridge, MA, USA

\section{REFERENCES}

1. Y. Xia et al., "Quantum-enhanced data classification with a variational entangled sensor network," Phys. Rev. X 11, 021047 (2021).

2. Y. Cao et al., "Quantum chemistry in the age of quantum computing," Chem. Rev. 119, 10856 (2019).

3. S. McArdle et al., "Quantum computational chemistry," arXiv:1808.10402.
4. V. Havlíček et al., "Supervised learning with quantum-enhanced feature spaces," Nature 567, 209 (2019).

5. J. Preskill, "Quantum Computing in the NISQ era and beyond," Quantum 2, 79 (2018).

6. C. W. Chou et al., "Frequency comparison of two high-accuracy $\mathrm{Al}^{+}$optical clocks," Phys. Rev. Lett. 104, 070802 (2010).

7. I. Chiorescu et al., "Magnetic strong coupling in a spin-photon system and transition to classical regime," Phys. Rev. B 82, 024413 (2010); D. I. Schuster et al., "High-cooperativity coupling of electron-spin ensembles to superconducting cavities," Phys. Rev. Lett. 105, 140501 (2010); Y. Kubo et al., "Strong coupling of a spin ensemble to a superconducting resonator," Phys. Rev. Lett. 105, 140502 (2010); H. Wu et al., "Storage of multiple coherent microwave excitations in an electron spin ensemble," Phys. Rev. Lett. 105, 140503 (2010). 